\title{
The role of genetics in neurodegenerative dementia: a large cohort study in South China
}

Bin Jiao ${ }^{1,2,3,4,5,9}$, Hui Liu ${ }^{1,9}$, Lina Guo ${ }^{1}$, Xuewen Xiao ${ }^{1}$, Xinxin Liao ${ }^{2,3,4,5,6}$, Yafang Zhou ${ }^{2,3,4,5,6}$, Ling Weng ${ }^{1,2,3,4,5}$, Lu Zhou ${ }^{1}$, Xin Wang ${ }^{1}$, Yaling Jiang ${ }^{1}$, Qijie Yang ${ }^{1}$, Yuan Zhu ${ }^{1}$, Lin Zhou ${ }^{2,5,7}$, Weiwei Zhang ${ }^{2,3,4,5,8}$, Junling Wang ${ }^{1,2,3,4,5}$, Xinxiang Yan ${ }^{1,2,3,4,5}$, Jinchen Li ${ }^{2,3,4,6}$, Beisha Tang $1,2,3,4,5$ and Lu Shen ${ }^{1,2,3,4,5,7 凶}$

Neurodegenerative dementias are a group of diseases with highly heterogeneous pathology and complicated etiology. There exist potential genetic component overlaps between different neurodegenerative dementias. Here, 1795 patients with neurodegenerative dementias from South China were enrolled, including 1592 with Alzheimer's disease (AD), 110 with frontotemporal dementia (FTD), and 93 with dementia with Lewy bodies (DLB). Genes targeted sequencing analysis were performed. According to the American College of Medical Genetics (ACMG) guidelines, 39 pathogenic/likely pathogenic (P/LP) variants were identified in 47 unrelated patients in 14 different genes, including PSEN1, PSEN2, APP, MAPT, GRN, CHCHD10, TBK1, VCP, HTRA1, OPTN, SQSTM1, SIGMAR1, and abnormal repeat expansions in C9orf72 and HTT. Overall, 33.3\% (13/39) of the variants were novel, the identified P/LP variants were seen in 2.2\% (35/1592) and 10.9\% (12/110) of AD and FTD cases, respectively. The overall molecular diagnostic rate was $2.6 \%$. Among them, PSEN1 was the most frequently mutated gene (46.8\%, 22/47), followed by PSEN2 and APP. Additionally, the age at onset of patients with P/LP variants (51.4 years), ranging from 30 to 83 years, was 10 years earlier than those without $\mathrm{P} / \mathrm{LP}$ variants $(p<0.05)$. This study sheds insight into the genetic spectrum and clinical manifestations of neurodegenerative dementias in South China, further expands the existing repertoire of P/LP variants involved in known dementiaassociated genes. It provides a new perspective for basic research on genetic pathogenesis and novel guiding for clinical practice of neurodegenerative dementia.

npj Genomic Medicine (2021)6:69; https://doi.org/10.1038/s41525-021-00235-3

\section{INTRODUCTION}

Neurodegenerative dementias are a group of clinically heterogeneous diseases with frequently overlapping symptoms, such as multi-cognitive impairments, behavioral changes, and movement deficits ${ }^{1}$. Alzheimer's disease (AD) is the most common dementia worldwide, accounting for $60-80 \%$ of all dementia cases ${ }^{2}$. Frontotemporal dementia (FTD) is the second most common cause of neurodegenerative dementia after $A D$ in patients younger than 65 years, responsible for $10.2 \%$ of cases $^{3}$, and dementia with Lewy bodies (DLB) has been reported as being the second most common dementia subtype in older people following $A D$, accounting for $7.5 \%$ of all dementia cases ${ }^{4}$. However, the etiology of neurodegenerative dementias is still obscure, which is thought to be caused by a combination of ageing, environmental, and genetic factors.

Recently, substantial progress has been made regarding the molecular genetics of neurodegenerative dementias. PSEN1, $P S E N 2$, and $A P P$ are recognized as three causative genes for familial $A D$ (FAD), which explains the genetic background of $5-10 \%$ of early onset $A D$ (EOAD, younger than 65 years). The estimated mutation frequencies of PSEN1, APP, and PSEN2 in EOAD, are $80 \%, 15 \%$, and $5 \%$, respectively ${ }^{5}$. Likewise, FTD is a genetically and pathologically heterogeneous disorder with a higher incidence of familial cases than AD. Genetic etiology has been revealed in $\sim 30-50 \%$ of FTD patients with a positive family history ${ }^{6,7}$. At present, more than 10 genes are related to FTD, and MAPT, GRN, and C9orf72 are the most common, accounting for $\sim 60 \%$ of all cases of inherited FTD $^{3}$. In contrast, the genetic architecture of DLB remains largely elusive ${ }^{8}$. To date, only three genes have been confirmed to be related to DLB, including $A P O E$, $G B A$, and SNCA. However, growing evidence supports that DLB has a strong and unique genetic component ${ }^{9}$.

Interestingly, previous studies have suggested a potential genetic overlap between AD, FTD, and DLB. Notably, PSEN1, the most common etiology of EOAD, has also been found in patients with FTD and DLB ${ }^{10-13}$. Similarly, mutations in MAPT, GRN, and C9orf72 have also been detected at lower frequencies in $A D$ and DLB patients ${ }^{14-16}$. Homozygosity for APOE4, the strongest genetic risk factor for $A D$, has also been reported in several studies to increase the risk of FTD and DLB ${ }^{17,18}$. In addition, mutations in SNCA have been shown to result in a wide phenotypic spectrum of DLB, Parkinson's disease (PD), multiple system atrophy (MSA), and FTD $^{19-21}$

In this study, we comprehensively analyzed the mutational spectrum of known dementia-associated genes from patients with neurodegenerative dementias in the South Chinese population using integrated targeted gene sequencing analysis. First, we systematically identified pathogenic and likely pathogenic (P/LP) variants of known dementia-associated genes, including known and novel variants, summarized and compared the mutation

\footnotetext{
${ }^{1}$ Department of Neurology, Xiangya Hospital, Central South University, Changsha, China. ${ }^{2}$ National Clinical Research Center for Geriatric Disorders, Central South University, Changsha, China. ${ }^{3}$ Engineering Research Center of Hunan Province in Cognitive Impairment Disorders, Central South University, Changsha, China. ${ }^{4}$ Hunan International Scientific and Technological Cooperation Base of Neurodegenerative and Neurogenetic Diseases, Changsha, China. ${ }^{5}$ Key Laboratory of Hunan Province in Neurodegenerative Disorders, Central South University, Changsha, China. ${ }^{6}$ Department of Geriatrics, Xiangya Hospital, Central South University, Changsha, China. ${ }^{7}$ Key Laboratory of Organ Injury, Aging and Regenerative Medicine of Hunan Province, Changsha, China. ${ }^{8}$ Department of Radiology, Xiangya Hospital, Central South University, Changsha, China. ${ }^{9}$ These authors contributed equally: Bin Jiao, Hui Liu. ${ }^{凶}$ email: shenlu@csu.edu.cn
} 
Table 1. Summary of clinical features of the cognitive impairment disease patients in this study.

\begin{tabular}{|c|c|c|c|c|}
\hline Clinical features & $\mathrm{AD}(n=1592)$ & FTD $(n=110)$ & $\operatorname{DLB}(n=93)$ & Total $(n=1795)$ \\
\hline Gender (M, \%) & $645,40.4 \%$ & $56,50.9 \%$ & $57,61.3 \%$ & $758,42.1 \%$ \\
\hline Family history $(+, \%)$ & $467,29.3 \%$ & $35,31.8 \%$ & $16,17.2 \%$ & $518,28.8 \%$ \\
\hline Disease duration, years & $3.0 \pm 2.4$ & $2.8 \pm 2.3$ & $2.9 \pm 2.8$ & $3.0 \pm 2.4$ \\
\hline \multicolumn{5}{|l|}{ APOE genotypes $(n, \%)$} \\
\hline APOE $4+/+$ & $145,9.1 \%$ & $5,4.5 \%$ & $7,7.5 \%$ & $157,8.7 \%$ \\
\hline APOE4 +I- & $561,35.2 \%$ & $36,32.7 \%$ & $29,31.2 \%$ & $626,34.9 \%$ \\
\hline APOE4 -/- & $886,55.7 \%$ & $69,62.8 \%$ & $57,61.3 \%$ & $1012,56.4 \%$ \\
\hline
\end{tabular}

The age at onset, the age at diagnosis, disease duration, educational attainment, MMSE scores are all shown as mean \pm standard deviation. Gender, family history, APOE genotypes are all shown as numbers and proportions (\%).

$A D$ Alzheimer's disease, FTD frontotemporal dementia, DLB dementia with Lewy bodies, MMSE Mini-Mental State Examination.

frequency among patients with different clinical diagnosis. Second, we generalized the clinical manifestation of neurodegenerative dementia patients carried $P / L P$ variants in this study, including PSEN1, PSEN2, APP, MAPT, GRN, C9orf72, CHCHD10, HTRA1, TBK1, OPTN, SQSTM1, VCP, SIGMAR1, and HTT, attempting to summarize the relationship between gene mutations and clinical phenotypes. Then, we compared the age at onset (AAO) of patients with and without P/LP variants and patients carried different genes separately, to depict the AAO spectrum for these dementia-associated genes in our population. Finally, we analyzed $A P O E$ genotypes (non-carriers or carriers of $A P O E 4$ ) in $A D$ cohort and conclude the difference between $A P O E$ genotypes and different $A D$ subgroups. Our studies provide a new perspective for further basic research of neurodegenerative dementia, especially genetic-associated pathogenesis and facilitated the clinical prediction, diagnosis, and genetic counseling.

\section{RESULTS}

\section{Demographics and analysis of genes targeted sequence}

In this study, 1592 AD patients, 110 FTD patients, and 93 DLB patients were included. Demographic and clinical characteristics are shown in Table 1. A total of $39 \mathrm{P} / \mathrm{LP}$ variants from 14 genes are identified in 47 unrelated patients by a dementia-related gene panel, which contained 36 genes associated with cognitive impairment phenotype (Supplementary Table 1 and Supplementary 2). Among them, $33.3 \%$ of variants (13/39) were novel, including PSEN1 (c.451G $>A$, c679A $>$ c, c.A1139>G, and c.1369 A>G), PSEN2 (c.T716C and c.1180delG), GRN (c.20G>A), CHCHD10 (c.121C>T and c.283C >T), OPTN (c.1402_1407del), SQSTM1 (c.558_559insC), SIGMAR1 (c.26G>A), and TBK1 (c.973dup). All identified P/LP variants were responsible for $2.2 \%$ of AD (35/1592) and $10.9 \%$ of FTD (12/110), which led to an overall molecular diagnostic yield of $2.6 \%$ (Fig. 1), however, in this study, no P/LP variants were identified in DLB patients. $70.2 \%$ (33/47) of patients had a positive family history and $46.8 \%$ (22/47) of patients with P/LP variants had at least one APOE4.

\section{Mutational spectrum of the AD cohort}

Overall, 27 different P/LP variants were identified in 35 unrelated $A D$ patients from five genes, including PSEN1, PSEN2, APP, CHCHD10, and HTRA1 (Table 2). 88.6\% (31/35) carrier were FAD probands, $11.4 \%(4 / 35)$ were sporadic AD (SAD) cases.

In this study, PSEN1 was the most frequently mutated gene, 19 $\mathrm{P} / \mathrm{LP}$ missense mutations were identified in 22 patients, among which four were novel identified in our study, including c.451G $>A$, p.V151M; c.679A >C, p.1227L; c.1139A >G, p.K380R; and c.1369A>G, p.M457V. Seven patients carried PSEN2 P/LP variants, including six missense mutations at the same amino acid residue (M239) and one frameshift mutation. Two were novel, including c.T716C, p.239M $>T$ and c.1180delG, p.A394Pfs*8. All patients with the variants had a positive family history except for one who carried PSEN2 p.239M >T. Meanwhile, two APP missense mutations were identified in four FAD probands, including c.2143G>A, p.V715M, and c.2149G $>A$, p.V717l (Table 2). The distribution of PSEN1/ PSEN2/APP P/LP variants are shown in Fig. 2. Interestingly, all identified $P / L P$ variants of PSEN1/PSEN2/APP were located in hydrophobic regions or in the endoproteolytic cleavage regions.

In patients with PSEN1/PSEN2/APP variants, 93.9\% (31/33) were defined as early-onset $A D(E O A D)(A A O<65$ years)), $45.5 \%$ were APOE4-negative, $48.5 \%$ had one APOE4, and $6.0 \%$ had two copies of APOE4.

About clinical phenotypes, all PSEN1/PSEN2/APP variants carriers initially presented with memory decline. Then, language impairment and behavior change were common symptoms in these variants, 52.63\% (10/19) PSEN1, 25\% (1/4) PSEN2, and 50\% (1/2) $A P P$ P/LP variants showed language impairment, respectively, such as naming difficulty, repetitive speech, fluency disorder, and speech reduction, while the frequency of mental and behavior change of PSEN1, PSEN2, APP variants were $57.6 \%$ (11/19), 75\% (3/ 4), and $100 \%(2 / 2)$, respectively. Meanwhile, $26.3 \%$ (5/19) PSEN1, 25\% (1/4) PSEN2 P/LP variants presented sensory and movement disorders, such as hallucination, delusion, weakness, involuntary movement, and abnormal gait. Interestingly, the clinical manifestation of patients with PSEN2 mutations at amino acid residue M239 showed high heterogeneity, including memory decline, language impairment, mental and behavior change, and sensory and movement disorders.

Additionally, we also found two female AD patients who carried a nonsense mutation in CHCHD10 (c.283C>T, p.Q95*) and HTRA1 (c.589C >T, p.R197*), respectively. The patient who carried CHCHD10 p.Q95* showed memory decline at 52 years and gradually developed language dysfunction, behavioral changes, bradykinesia, and depression. Brain MRI showed bilateral atrophy of temporal parietal lobe and hippocampus, and cerebrospinal fluid (CSF) examination showed the level of $A \beta 42$ and $A \beta 42 / A \beta 40$ ratio decreased, while the phospho-tau ( $\mathrm{p}$-tau) and total tau (t-tau) increased. In addition, the Pittsburgh compound B (PiB)-PET showed diffuse amyloid deposition in the whole brain cortex. The patient who carried HTRA1, p.R197*, mainly presented typical 


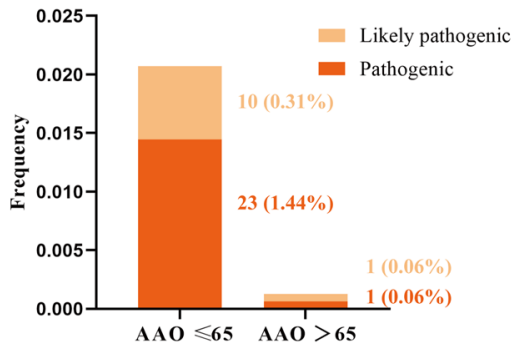

b

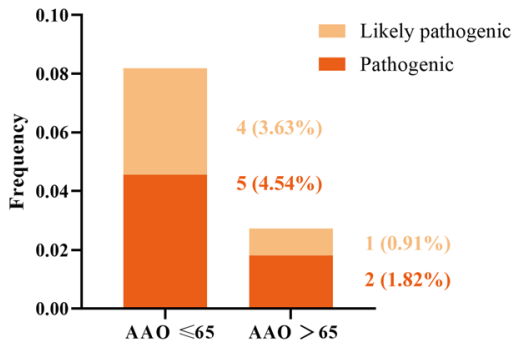

c

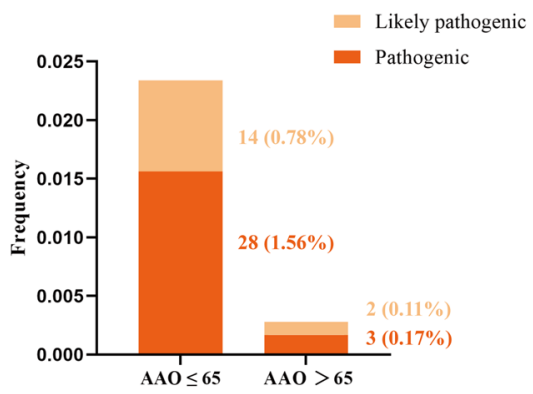

AD cohort $(n=1592)$

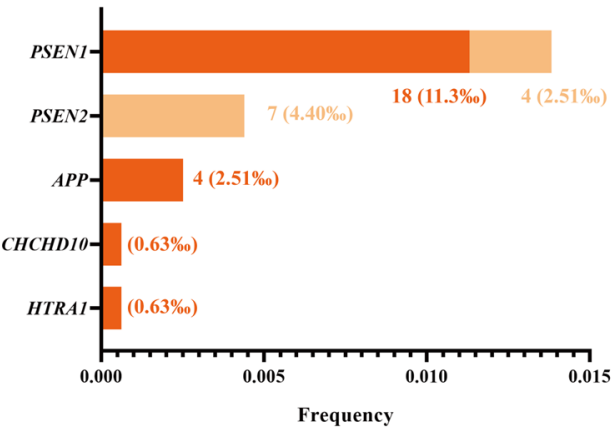

FTD cohort $(\mathbf{n}=110)$

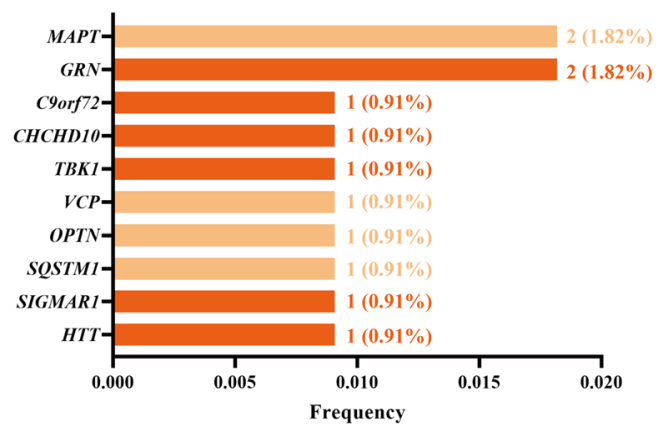

Entire cohort $(n=1795)$

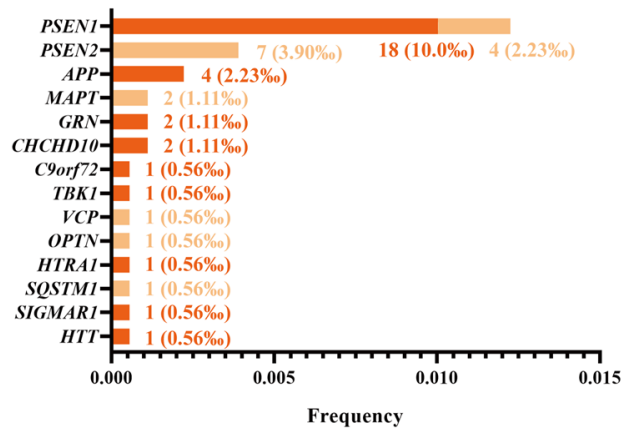

Fig. 1 Mutational frequencies of known cognitive impairment disease-associated genes in AD, FTD, and entire cohorts respectively. Mutational frequencies of all (left) and each (right) known cognitive impairment-associated genes in the different dementia cohorts. a AD cohort. b FTD cohort. c entire cohort. Variants that were classified as pathogenic or likely pathogenic according to the standards and guidelines of the ACMG. 'Pathogenic' means that the patients had pathogenic variants in known cognitive impairment disease-associated genes, and 'likely pathogenic' means that the patients had likely pathogenic variants in known cognitive impairment disease-associated genes. ACMG American College of Medical Genetics, AD Alzheimer's disease, FTD frontotemporal dementia.

forgetfulness of recent events and daily living ability declined at 49 years. Brain MRI showed multiple spot-like hyperintensities in the deep bilateral frontotemporal lobes and paraventricular region, while no microbleeds on susceptibility-weighted images sequence. The level of $A \beta 42$ in CSF decreased, and p-tau increased which supported the diagnosis of $A D$.

\section{Mutational spectrum of the FTD cohort}

A total of $12 \mathrm{P} / \mathrm{LP}$ variants in 10 genes were identified in the FTD cohort, including MAPT, GRN, C9orf72, CHCHD10, TBK1, OPTN, SQSTM1, VCP, SIGMAR1, and HTT, summarized in Table 2. Six were novel variants, including three nonsense mutations (GRN: c. 20G >A, p.W7*; CHCHD10: c. 121C>T, p.Q41*; and SIGMAR1: c.26G $>$ A, p.W9*), two frameshift mutations (TBK1: c.973dup, p. Y325Lfs*4 and SQSTM1: c.558_559insC, p.V287Rfs*21), and one deletion mutation (OPTN: c.1402_1407del, p.468_469del). Interestingly, only two P/LP variants carriers had a positive family history. The mean \pm SD AAO of P/LP variants carriers was $59.2 \pm$ 10.5 years, which was significantly older than $\mathrm{P} / \mathrm{LP}$ variants carriers in $\mathrm{AD}$ cohort $(48.8 \pm 7.7, p=0.001)$. Meanwhile, only three patients carried APOE4 $(25 \%, 3 / 12)$, which tended to be lower than P/LP variant carriers in the $A D$ cohort $(54.3 \%, p=0.077)$.

As for clinical characteristics, all patients carried P/LP variants in FTD cohort showed memory decline, $58.33 \%$ (7/12) patients had language impairment, mental, and behavior changes, and 25\% (4/ 12) $P / L P$ variants carriers accompanied by sensory and movement disorders. Interestingly, one showed personality changes and language impairment, as well as abnormal emotional responses at baseline. In the fifth year of onset, she suffered from memory decline. Brain MRI showed bilateral frontal lobe atrophy, and bvFTD was initially considered. However, molecular testing revealed that she carried heterozygous CAG expanded repeats in $H T$, which supported the diagnosis of Huntington's disease (HD). 


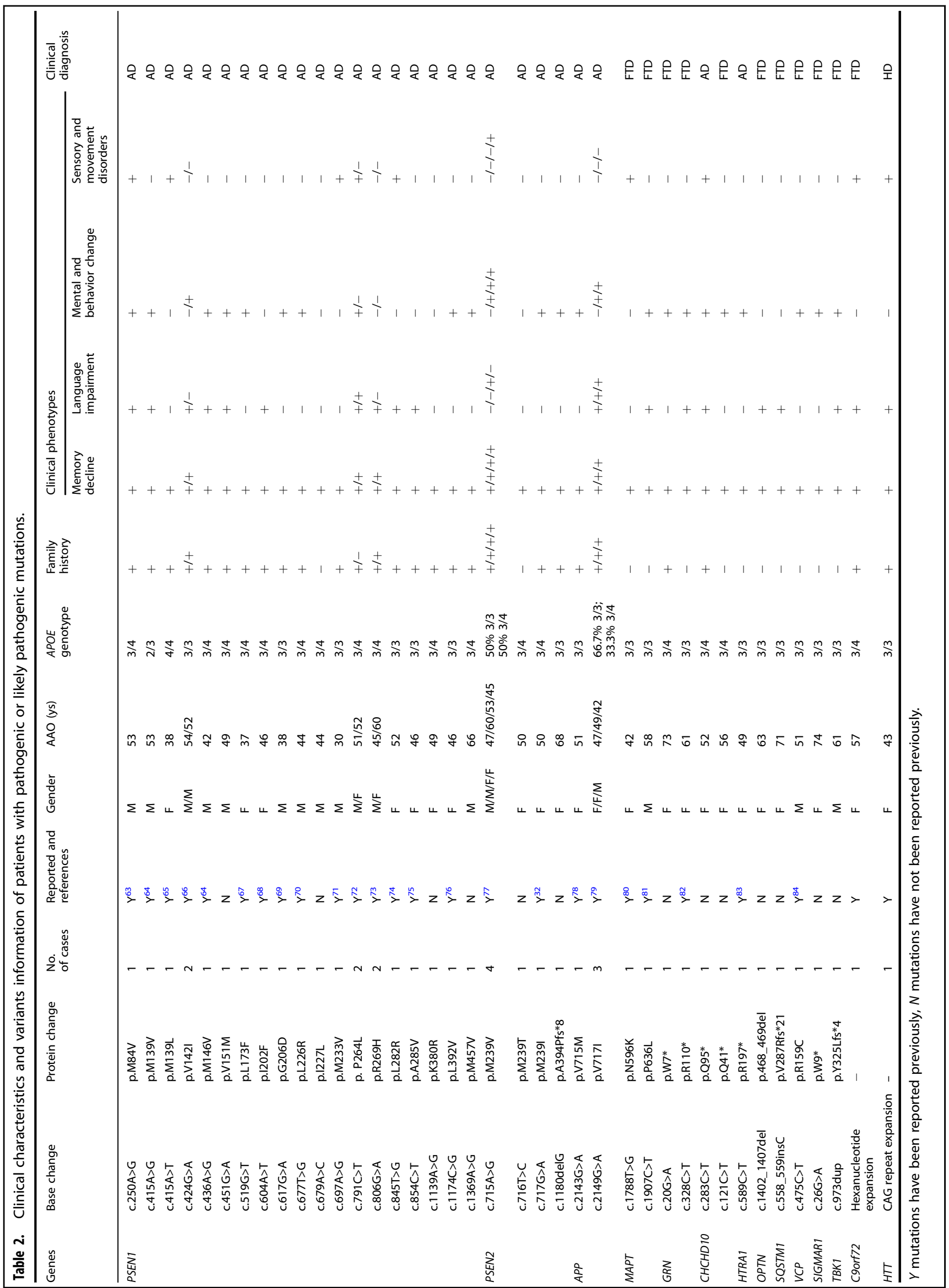




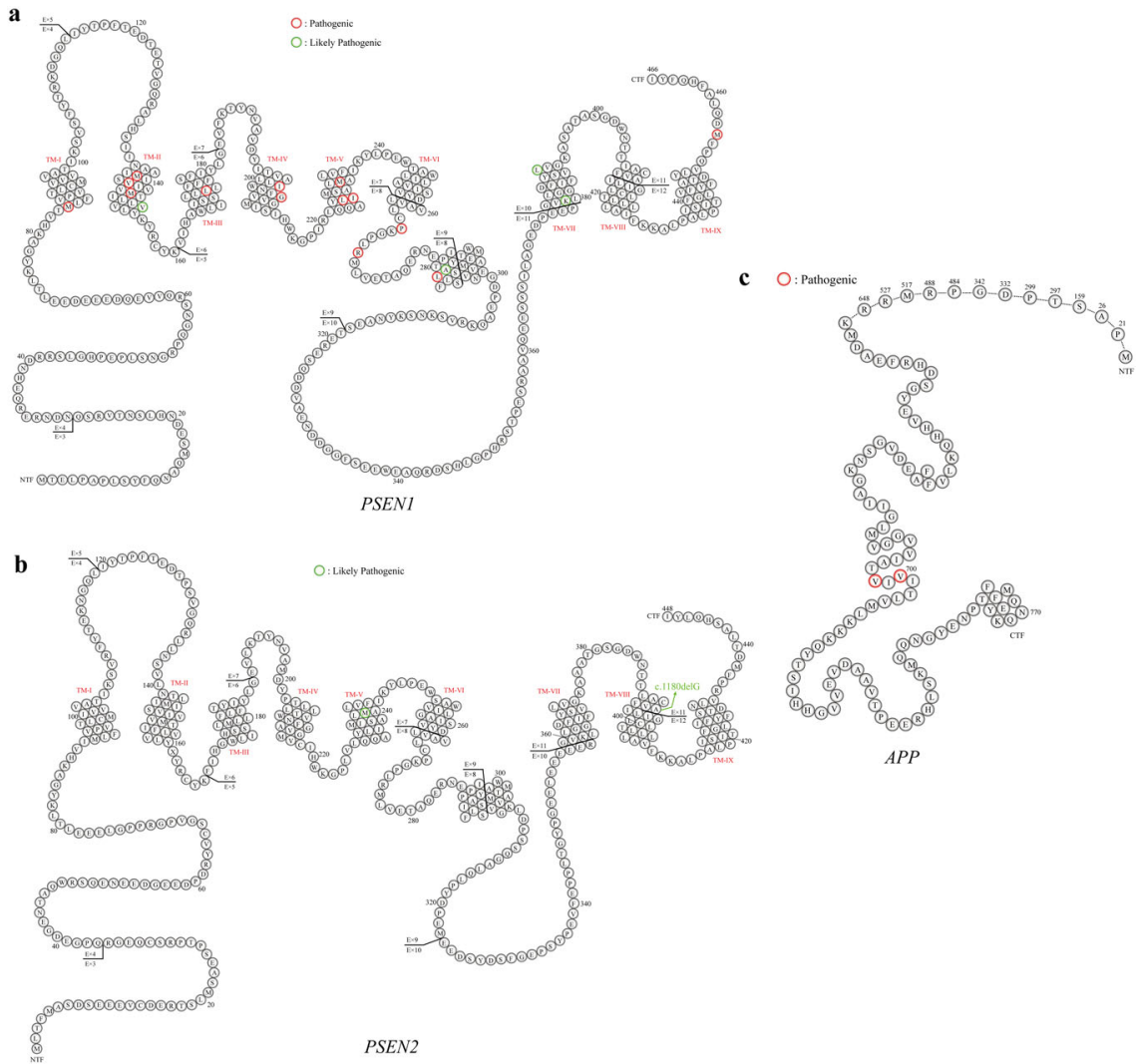

Fig. 2 Distribution of amino acid substitutions in the PSEN1, PSEN2, and APP proteins. Red circles: pathogenic mutations identified. Green circles: likely pathogenic mutations identified. A Ala, C Cys, D Asp, E Glu, F Phe, G Gly, H His, I lle, K Lys, L Leu, M Met, N Asn, P Pro, Q Gln, R Arg, S Ser, T Thr, V Val, W Trp, Y Tyr, APP amyloid precursor protein, PSEN presenilin. a The distribution of PSEN1 P/LP variants. $\mathbf{b}$ The distribution of PSEN2 P/LP variants. c The distribution of APP P/LP variatns.

\section{Spectrum of age at onset}

Moreover, the mean AAO were significantly younger in patients with P/LP variants in the AD cohort and entire cohort, while no difference was found in the FTD cohort (Fig. 3a-c). Specifically, the mean AAO of patients with P/LP variants in the entire cohort was $51.4 \pm 9.5$ years, $\sim 10$ years younger than the mean AAO of those without P/LP variants $(64.8 \pm 10.7$ years) $(p<0.001)$, among them, $89.4 \%$ were younger than 65 years.

Meanwhile, we analyzed the spectrum of AAO in patients with P/LP variants of different genes (genes with two or more mutations were included). The results showed that the mean AAO of subjects with P/LP variants of PSEN1, PSEN2, and APP $(47.5 \pm 10.7$ years, $53.2 \pm 8.1$ years, and $46.5 \pm 4.2$ years, respectively) were significantly lower than those of non-carriers $(p<0.05)$ (Fig. 3d).

In addition, we performed subgroup analysis on the family history and the status of APOE4 to compare the difference in AAO between the two groups respectively, which showed that the AAO of FAD patients was significantly younger than that of SAD $(63.2 \pm$ 11.4 and $64.9 \pm 10.6$, respectively, $p=0.005)$, while no significant between APOE4 carriers and non-carriers $(p=0.953)$ (Fig. 3e, f).

To analyze the confounding factors affecting AAO, we conducted further multiple linear regression analysis with AAO as the dependent variable. After controlling independent variables, including gender, disease duration, educational attainment, $A P O E$ genotypes, dementia family history, MMSE scores, mutation status, and clinical diagnosis, the model showed that MMSE scores $(B=-0.135, p<0.001)$, disease duration $(B=-0.421, p=0.001)$, and status of mutation carried $(B=-13.44, p<0.001)$.

\section{Characteristics of APOE genotypes}

As for the distributions of $A P O E$ genotypes, there was no significant difference across AD, FTD, and DLB cohorts $(p>$ 0.0166; Bonferroni corrected). Furthermore, APOE4 as the strongest genetic risk factor for $A D$, we further compared the distribution difference between EOAD and LOAD patients, FAD and SAD. We found no significant difference in APOE4 frequency (EOAD vs LOAD: $p=0.501$; FAD vs SAD: $p=0.153$, respectively). Further subgroup analysis in $A D$ cohort showed that the proportion of APOE4-negative patients was higher than that of APOE4-positive patients $(p<0.001, p=0.007$, and $p<0.001$, respectively) (Fig. $4 a, b$ ). In addition, we found no significant difference in the distribution difference of APOE4 between variants carriers and non-carriers in the $\operatorname{AD}$ cohort $(p=0.281)$. Meanwhile, a higher percentage of APOE4 ( + ) patients was found in $\mathrm{P} / \mathrm{LP}$ variants than in patients without $\mathrm{P} / \mathrm{LP}$ variant in $\mathrm{AD}$ cohort $(p<0.001)$ (Fig. 4c).

\section{DISCUSSION}

In this study, we determined the mutational spectrum of 36 known dementia-associated genes in patients clinically diagnosed with neurodegenerative dementia patients, including $A D, F T D$, and DLB in a South China population sample using integrated targeted gene sequencing analysis. This is the first report of distributions of gene mutations in patients with neurodegenerative dementias from South China. We observed that the use of an integrated gene analysis could be an effective tool for detecting potential genetic causes in neurodegenerative dementias with high genetic heterogeneity or overlapping phenotypic features, 
a

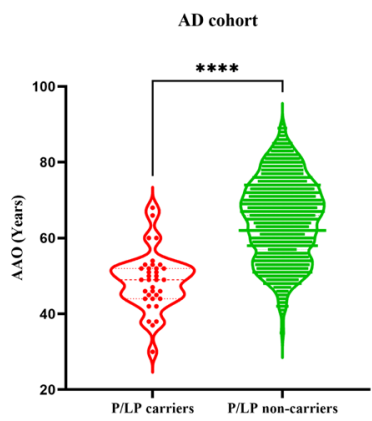

b

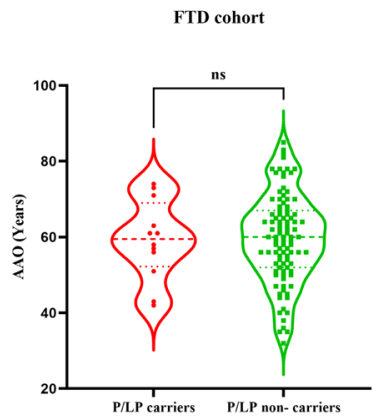

c

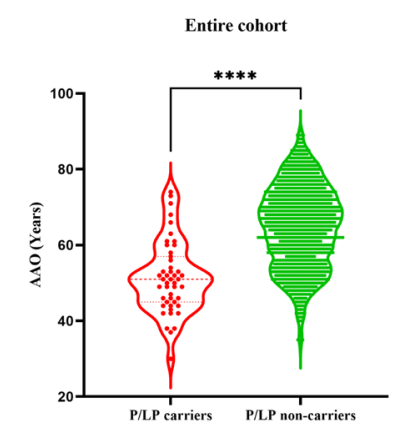

d

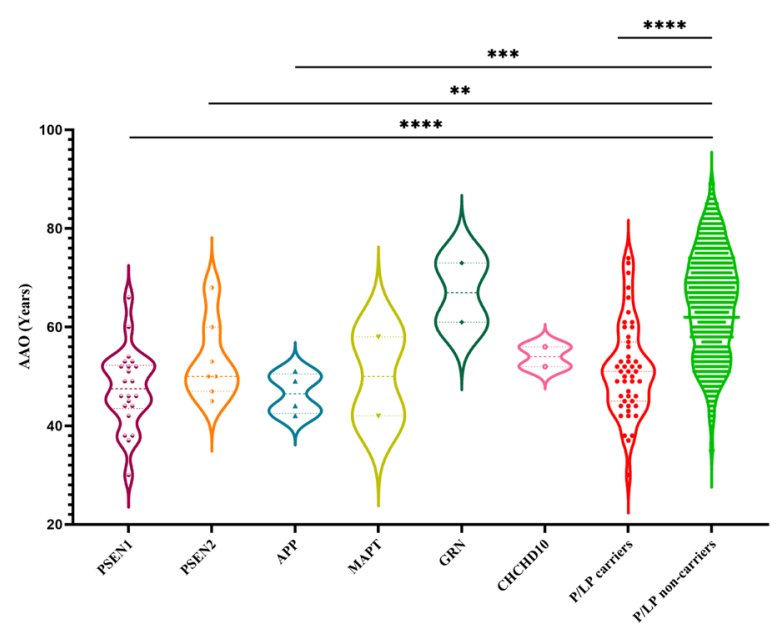

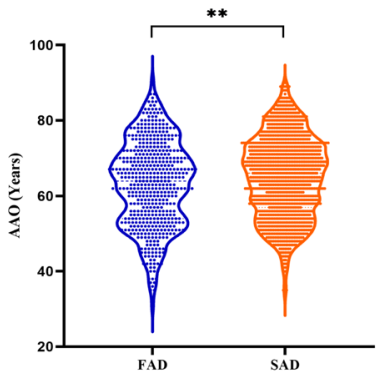

f

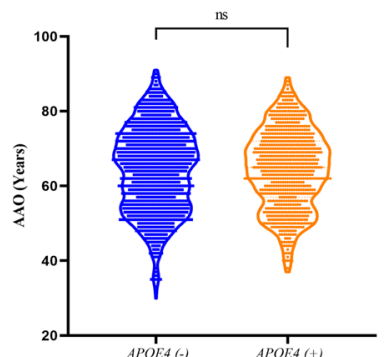

Fig. 3 AAO spectrum of known cognitive impairment disease-associated genes in AD, FTD, and entire cohorts. Comparison of AAO in all patients with P/LP variants of known cognitive impairment disease-associated genes and patients without P/LP variants in known cognitive impairment disease-associated genes. a AD cohort. b FTD cohort. $\mathbf{c}$ Entire cohort. The dashed red line refers to the mean AAO of patients with $\mathrm{P} / \mathrm{LP}$ variants in the corresponding cohorts, whereas the dashed line refers to the mean AAO of patients without P/LP variants in known cognitive impairment disease-associated genes in the corresponding cohort. d Spectrum of AAO in patients with P/LP variants of each cognitive impairment disease-associated gene (only genes carried by two or more patients were included), in patients with and without P/LP variants of known cognitive impairment disease-associated genes. e Spectrum of AAO in FAD and SAD patients. $f$ Spectrum of AAO in patients with and without APOE4. ${ }^{*} p<0.05,{ }^{* *} p<0.01,{ }^{* * *} p<0.001,{ }^{* * * *} p<0.0001$, ns no significance. AAO age at onset, AD Alzheimer's disease, FTD frontotemporal dementia, P/LP pathogenic or likely pathogenic, FAD familial Alzheimer's disease, SAD sporadic Alzheimer's disease.

gaining further insight in genetic pathogenesis of neurodegenerative dementia, clinical diagnosis, and genetic counseling.

Mutations in PSEN1 are the most common cause of EOAD, meanwhile, PSEN1 was the most frequently mutated gene in patients with FAD. To date, more than 300 mutations in PSEN1 have been identified to be associated with FAD. In this study, four novel variants were identified, which expanded the mutational spectrum of PSEN1. The AAO of PSEN1 mutation carriers in our study (47.5 years), was older than Ryan et al. reported (43.6 years) in $168 \mathrm{AD}$ patients with PSEN1 mutations ${ }^{22}$, but younger than Jia et al. reported in a large FAD cohort from China (50.59 years) ${ }^{23}$. Of interest, in addition to the PSEN1 mutations mentioned above, we found an older female (83 years) carrying a novel mutation (M270L), and the APOE genotype was $3 / 4$ in the DLB cohort. Several algorithms predicted the variant was not disease damaging, whereas the nearby mutations (R269G, R269H, and L271V) have been reported to be associated with $F A D^{24-26}$. Whether the clinical phenotype of the patient is caused by the novel mutation or the contribution of $A P O E$ genotype is unclear; we will perform functional research to further clarify the variant. Meanwhile, regarding clinical phenotypes, PSEN1 mutation carriers often present with atypical cognitive symptoms and additional neurological features ${ }^{22}$. However, in this study, patients mainly presented with amnesia, language impairment, mental and behavioral changes, and movement disorders, which is one limitation of this study. This might have two explanations. First, some atypical symptoms might not occur at an early stage of the disease, and follow-up is necessary. Second, the mutation locations may lead to distinguishing phenotypes; for example, atypical cognitive presentations and pyramidal signs were seen more frequently in association with PSEN1 mutations involving exon $8^{22}$, suggesting that multiple factors could contribute to the phenotypic heterogeneity of PSEN1-related AD.

In contrast to PSEN1, only 18 pathogenic mutations within PSEN2 have been reported, most of which occurred in European and African populations. In this study, seven P/LP variants were identified, including six FAD cases. Previous studies showed that the AAO of PSEN2-associated cases vary widely, from 45 to 88 


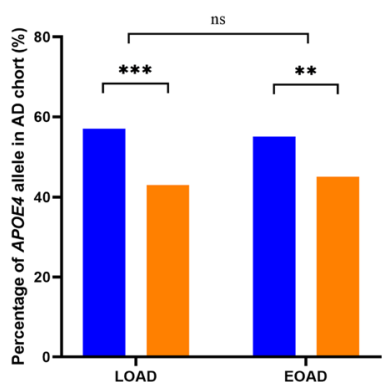

b

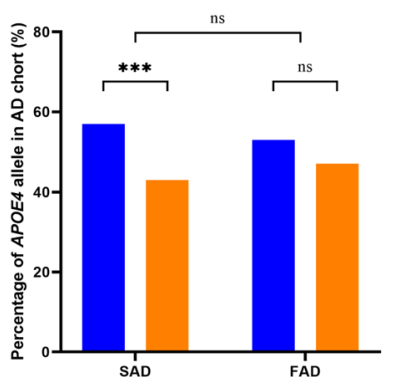

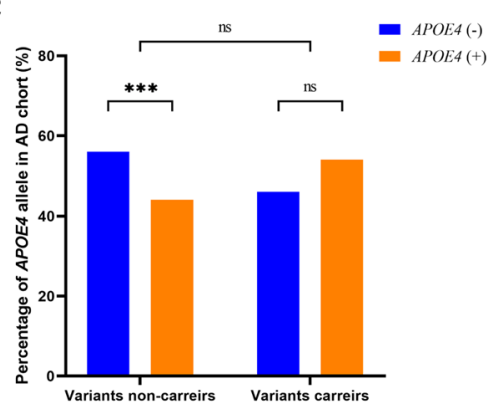

Fig. 4 The percentage of APOE4 in AD cohort. a The percentage of APOE4 in LOAD and EOAD. $\mathbf{b}$ The percentage of APOE4 in SAD and FAD. c The percentage of $A P O E 4$ in AD patients with and without P/LP variants. ${ }^{*} p<0.05,{ }^{* *} p<0.01,{ }^{* * *} p<0.001,{ }^{* * * *} p<0.0001$, ns no significance. LOAD late-onset Alzheimer's disease, EOAD early-onset Alzheimer's disease, SAD sporadic Alzheimer's disease, FAD familial Alzheimer's disease.

years; that is more than 10 years later than the mean AAO for PSEN1-related cases ${ }^{27-29}$, which was consistent with our results. Interestingly, six patients with a substitution at PSEN2 amino acid residue 239 were identified, including M239V, M239l, and M239T. Among them, M239V has been reported in European populations to elevate $A \beta 42$ levels and $A \beta 42 / A \beta 40$, and to exhibit a partial loss of function with respect to the C-terminal fragment- $\gamma$ as well as a substantial decrease in $A \beta 40$ levels ${ }^{30-32}$, but it has been absent from Asian cohorts so far. Our findings suggest that this residue may be a common causative variant in the South Chinese population. In addition, the clinical phenotypes of carriers of the M239V mutation varied widely. Our findings, together with previous reports, further suggest that phenotypic heterogeneity exists even at the same codon site because of different amino acid transversions ${ }^{30,32,33}$.

In accordance with other populations, the common mutation site of APP were residues 715 and 717. Taken together with our previous reports, our team have found four families carrying mutations at this site ${ }^{34}$. Amino acid residues 715 and 717 are located near the $\gamma$-secretase cleavage site, and mutation at this site may increase the hydrophobicity of the APP TM domain to anchor the protein within the membrane and elevate the $A \beta 42$ / $A \beta 40$ ratio $^{35,36}$. Interestingly, patients with mutations at this site often have non-memory symptoms, which can be misdiagnosed as FTD, because the behavioral problems occur earlier than the memory deficits. Totally, in all PSEN1/PSEN2/APP P/LP variants in LOAD, PSEN1 M457V, and PSEN2 A394Pfs*8 were novel variants, further functional validation was necessary and warranted.

In addition, we identified $C H C H D 10$ and HTRA 1 mutations in the $A D$ cohort. $C H C H D 10$ has been identified to be associated with a large spectrum of diseases, including FTD, ALS, AD, cerebellar ataxia, mitochondrial myopathy, late-onset spinal motor neuronopathy, and Charcot-Marie-Tooth disease type $2^{37-40}$. Previously, we have reported a late-onset $A D$ patient with the $C H C H D 10$ mutation $^{41}$. A homozygous HTRA1 mutation was known to be causative for CARASIL ${ }^{42}$, while evidence was also showed that heterozygous HTRA1 mutation, which might result in an impaired HTRA1 activation cascade or be unable to form stable trimers, is related to autosomal dominant hereditary cerebral small vessel disease with delayed onset ${ }^{43-45}$. In this study, the female $A D$ patients presented with typical amnesia symptoms at 49 years without any other neurological or extra-neurological symptoms. Meanwhile, the Fazekas score of periventricular white matter hyperintensities was 1 , the APOE genotype was $3 / 3$ and the core biomarkers of CSF showed $\mathrm{A}+\mathrm{T}+\mathrm{N}$-. However, the effect of the heterozygous mutation in the pathophysiologic process of $A D$ remains elusive; further functional studies are still needed. These results further indicate that mutations not only in PSEN1, PSEN2, and $A P P$ can cause the AD phenotype, but that variants in other genes might also cause AD-like symptoms. Further follow-up is necessary.

Notably, we identified double mutations in a 52-year-old female (PSEN2 p.M239l and MAPT p.R5H), but her daily living ability remained intact, and the double mutation did not accelerate the cognitive decline, further expanding the phenotype spectrum of the mutation and supporting the phenotypic heterogeneity among subjects carrying the same MAPT mutations. Further in vivo and in vitro studies are needed to determine the effect of MAPT and PSEN2 mutations on the pathology and pathogenesis of AD.

Many different gene are reported to cause FTD, of which MAPT, GRN, and C9orf72 are three most common ${ }^{46-48}$. Except of three common genes, we also found variants in another seven genes, including CHCHD10, OPTN, SQSTM1, VCP, SIGMAR1, TBK1, and $H T^{49-52}$. In addition to genetics, the clinical phenotypes of FTD are also highly heterogeneous. In this study, we did not observe the classic phenotypes of mutations in $V C P$, such as inclusion body myopathy with Paget's disease of the bone $e^{53,54}$, we will follow up the patient to see the symptoms evolve. Moreover, the wrong diagnosis of the patient carried heterozygous CAG expanded repeats in $H T T$, further indicated that the overlap of clinical phenotypes is one of the main reasons for the difficulty in the diagnosis of neurodegenerative diseases. Genetic analysis is an effective method to improve diagnostic certainty.

Additionally, in our study, the proportion of APOE4 positive cases between FAD and SAD, EOAD and LOAD, were not significantly different, which is inconsistent with previous studies reporting that $A P O E 4$ exerts its maximal effect in $E O A D^{55,56}$. Perhaps other genetic or environmental factors may play an important role in the onset and pathogenesis of $A D$.

This study represents a comprehensive and systematic screening of 36 dementia-associated genes in AD, FTD, and DLB patients from South China, although the current study has some limitations. First, we only focused on known 36 dementiaassociated genes, not susceptibility genes, risk loci, or new candidate genes, which may play important roles in neurodegenerative dementia. Second, in this study, we only screened neurodegenerative dementia patients, but no controls were assessed to compare background frequencies of the P/LP variants. Lastly, for those novel variants identified in this study, we did not design functional experiments to further validation.

In conclusion, we have conducted the most systematic survey of the mutational spectrum of neurodegenerative dementia patients in South Chinese population, which further expanded the mutational spectrum of dementia-related genes and have provided evidence that there is some genetic heterogeneity and perhaps overlap between phenotypes. Our results may prove to be beneficial for clinical prediction, diagnosis, and genetic counseling and may generate hypotheses for future basic 


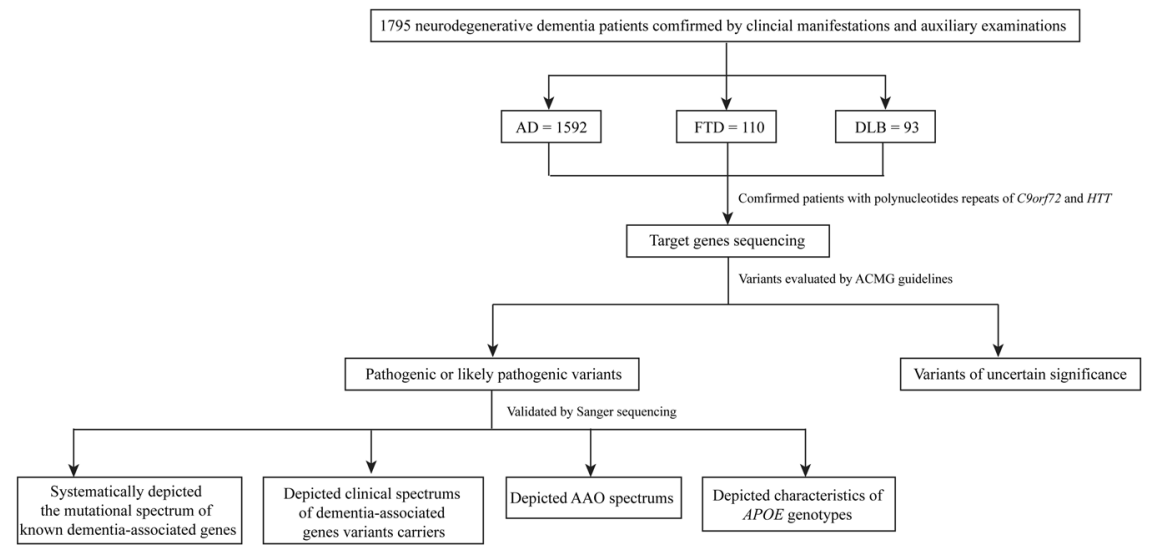

Fig. 5 Workflow of this study. AD Alzheimer's disease, FTD frontotemporal dementia, DLB dementia with Lewy bodies.

research on genetic-associated pathogenesis of neurodegenerative dementia.

\section{METHODS}

\section{Study participants}

A total of 1795 patients with neurodegenerative dementias, including 1592 with $A D, 110$ with FTD, and 93 with DLB, were recruited at the Xiangya Hospital, Central South University, between February 2004 and October 2020. All patients were unrelated probands. The demographic and clinical characteristics are summarized in Table 1 . All subjects had been clinically diagnosed with $A D, F T D$, or DLB according to international guidelines. This study was approved by the Ethics Committee of Xiangya Hospital, Central South University, China. Written informed consent was obtained from each participant or guardian.

\section{Targeted genes sequencing}

Genomic DNA was extracted from peripheral blood leukocytes of each participant using the QIAGEN kit according to the manufacturer's instructions. We designed a dementia-related gene panel containing a total of 36 genes associated with cognitive impairment phenotypes, including PSEN1, PSEN2, APP, APOE, ABCA7, SORL1, TREM2, ADAM10, MAPT, GRN, FUS, TARDBP, VCP, TBK1, CHCHD10, HTRA1, SQSTM1, UBQLN1, CHMP2B, SIGMAR1, OPTN, HNRNPA1, HNRNPA2B1, PRKAR1B, TMEM106B, UBQLN2, NOTCH3, TREX1, GLA, COL4A1, CSF1R, GBA, SNCA, SNCB, LRRK2, and PRNP. Briefly, gDNA was fragmented and a paired-end library was constructed using Covaris LE220 (Massachusetts, USA), followed by pre-capture PCR amplification. After PCR amplification, the DNA fragments were captured by the targeted panel, followed by sequencing using the Illumina NovaSeq 6000 platform. The reads were mapped to the human genome reference (hg19) using the Burrows Wheeler Aligner software (http://bio-bwa. sourceforge.net) ${ }^{57}$, and duplicate sequence reads were removed using Picard (http://broadinstitute.github.io/picard/). Variant calling was performed using the Genome Analysis Toolkit (https://software.broadinstitute. org/gatk/) ${ }^{58}$. The variants were annotated using ANNOVAR (https://hpc.nih. gov/apps/ANNOVAR.html ${ }^{59}$ and named according to the guidelines of the Human Genome Variation Society (http://www.hgvs.org/ ${ }^{60}$. Pathogenic or likely pathogenic (P/LP) variants were assessed according to the guidelines issued by the American College of Medical Genetics (ACMG).

Moreover, the (GGGGCC)n repeats in C9orf72 and (CAG)n repeats in HTT were performed in all individuals using previously reported repeat-primed polymerase chain reaction and capillary electrophoresis ${ }^{61,62}$.

\section{Sanger sequencing}

All $\mathrm{P} / \mathrm{LP}$ variants were estimated by $\mathrm{PCR}$ amplification and Sanger sequencing using a Big Dye Terminator V3.1 on an ABI 3730xI DNA analyzer (Applied Biosystems, Foster City, USA). The DNA sequences were then analyzed using Sequencher software version 4.2. All primers were designed using Primer 5, and the primer sequences and PCR reaction conditions are listed in Supplementary Table 3. Meanwhile, variants of unknown significance identified in this study are shown in Supplementary Table 4. The study workflow is shown in Fig. 5.

\section{Statistical analysis}

Quantitative variables such as age at onset, age at diagnosis, disease duration, education attainment, and cognitive assessment score are expressed as the mean $\pm S D$. All data were tested for normality and homogeneity of variance using the Shapiro-Wilk test and Levene variance equality test. Two independent samples were conducted using the $t$ test or the Mann-Whitney $U$ test. The $X^{2}$ test and Fisher exact test were used to analyze categorical data, such as the proportion of female patients, family history, the percentage of APOE4 positive or negative, and proportion of EOAD, LOAD or P/LP variants carriers and non-carriers patients. Multiple linear regression analysis was performed to correct the confounding factors and explore the factors affecting the AAO. All tests were two-tailed, and $p<$ 0.05 was considered statistically significant. All analyses were performed using SPSS v.26 (IBM). Data were visualized using Prism 8 (GraphPad).

\section{Reporting summary}

Further information on research design is available in the Nature Research Reporting Summary linked to this article.

\section{DATA AVAILABILITY}

The sequencing raw data analyzed during this study has been deposited in European Variation Archive, the accession number was PRJEB46658. All other data are available from the corresponding authors on reasonable request.

\section{CODE AVAILABILITY}

No custom code or scripts were used for the generation or processing of datasets.

Received: 28 March 2021; Accepted: 30 July 2021;

Published online: 13 August 2021

\section{REFERENCES}

1. Jack, C. R. et al. Prevalence of biologically vs clinically defined alzheimer spectrum entities using the national institute on aging-Alzheimer's association research framework. JAMA Neurol. 76, 1174 (2019).

2. Alzheimer's Association. 2020 Alzheimer's disease facts and figures. Alzheimers Dement. 16, 391-460 (2020).

3. Van Mossevelde, S., Engelborghs, S., van der Zee, J. \& Van Broeckhoven, C. Genotype-phenotype links in frontotemporal lobar degeneration. Nat. Rev. Neurol. 14, 363-378 (2018).

4. Vann, J. S. \& O'Brien, J. T. The prevalence and incidence of dementia with Lewy bodies: a systematic review of population and clinical studies. Psychol. Med. 44, 673-683 (2014).

5. Hinz, F. I. \& Geschwind, D. H. Molecular genetics of neurodegenerative dementias. Cold Spring Harb. Perspect. Biol. 9, a023705 (2017).

6. Rademakers, R., Neumann, M. \& Mackenzie, I. R. Advances in understanding the molecular basis of frontotemporal dementia. Nat. Rev. Neurol. 8, 423-434 (2012).

7. Rohrer, J. D. et al. The heritability and genetics of frontotemporal lobar degeneration. Neurology 73, 1451-1456 (2009). 
8. Erkkinen, M. G., Kim, M. O. \& Geschwind, M. D. Clinical neurology and epidemiology of the major neurodegenerative diseases. Cold Spring Harb. Perspect. Biol. 10, a033118 (2018).

9. Guerreiro, R. et al. Investigating the genetic architecture of dementia with Lewy bodies: a two-stage genome-wide association study. Lancet Neurol. 17, 64-74 (2018).

10. Bernardi, L. et al. Novel PSEN1 and PGRN mutations in early-onset familial frontotemporal dementia. Neurobiol. Aging 30, 1825-1833 (2009).

11. Ishikawa, A. et al. A mutantPSEN1 causes dementia with lewy bodies and variant Alzheimer's disease. Ann. Neurol. 57, 429-434 (2005).

12. Geiger, J. T. et al. Next-generation sequencing reveals substantial genetic contribution to dementia with Lewy bodies. Neurobiol. Dis. 94, 55-62 (2016).

13. Raux, G. et al. Dementia with prominent frontotemporal features associated with L113P presenilin 1 mutation. Neurology 55, 1577-1578 (2000).

14. Kelley, B. J. et al. Alzheimer disease-like phenotype associated with the c.154delA mutation in progranulin. Arch. Neurol. 67, 171-177 (2010).

15. Coppola, G. et al. Evidence for a role of the rare p.A152T variant in MAPT in increasing the risk for FTD-spectrum and Alzheimer's diseases. Hum. Mol. Genet. 21, 3500-3512 (2012).

16. Orme, T. et al. Analysis of neurodegenerative disease-causing genes in dementia with Lewy bodies. Acta Neuropathol. Commun. 8, 5 (2020).

17. Mishra, A. et al. Gene-based association studies report genetic links for clinical subtypes of frontotemporal dementia. Brain 140, 1437-1446 (2017).

18. Tsuang, D. et al. APOE epsilon4 increases risk for dementia in pure synucleinopathies. JAMA Neurol. 70, 223-228 (2013).

19. Pasanen, $P$. et al. A novel a-synuclein mutation A53E associated with atypical multiple system atrophy and Parkinson's disease-type pathology. Neurobiol. Aging 35, 2180.e1-2180.e5 (2014).

20. Markopoulou, K. et al. Clinical, neuropathological and genotypic variability in SNCA A53T familial Parkinson's disease: Variability in familial Parkinson's disease. Acta Neuropathol. 116, 25-35 (2008).

21. Bougea, A. et al. Frontotemporal dementia as the presenting phenotype of $p$. A53T mutation carriers in the alpha-synuclein gene. Parkinsonism Relat. Disord. 35, 82-87 (2017).

22. Ryan, N. S. et al. Clinical phenotype and genetic associations in autosomal dominant familial Alzheimer's disease: a case series. Lancet Neurol. 15, 1326-1335 (2016).

23. Jia, L. et al. PSEN1, PSEN2, and APP mutations in 404 Chinese pedigrees with familial Alzheimer's disease. Alzheimers Dement. 16, 178-191 (2020).

24. Perez-Tur, J. et al. A further presenilin 1 mutation in the exon 8 cluster in familial Alzheimer's disease. Neurodegeneration 5, 207-212 (1996).

25. Gómez-Isla, T. et al. A novel presenilin-1 mutation increased beta-amyloid and neurofibrillary changes. Ann. Neurol. 41, 809-813 (1997).

26. Kwok, J. B. J. et al. Presenilin-1 mutation L271V results in altered exon 8 splicing and Alzheimer's disease with non-cored plaques and no neuritic dystrophy. $J$. Biol. Chem. 278, 6748-6754 (2003).

27. Bird, T. D. et al. Wide range in age of onset for chromosome 1-related familial Alzheimer's disease. Ann. Neurol. 40, 932-936 (1996).

28. Sherrington, R. Alzheimer's disease associated with mutations in presenilin 2 is rare and variably penetrant. Hum. Mol. Genet. 5, 985-988 (1996).

29. Jiang, H., Jayadev, S., Lardelli, M. \& Newman, M. A review of the familial Alzheimer's disease locus PRESENILIN 2 and its relationship to PRESENILIN 1. J. Alzheimers Dis. 66, 1323-1339 (2018)

30. Lin, C. et al. Familial Alzheimer's disease in kindreds with missense mutations in a gene on chromosome 1 related to the Alzheimer's disease type 3 gene. Nature 376, 775-778 (1995).

31. Walker, E. S., Martinez, M., Brunkan, A. L. \& Goate, A. Presenilin 2 familial Alzheimer's disease mutations result in partial loss of function and dramatic changes in Abeta 42/40 ratios. J. Neurochem. 92, 294-301 (2005).

32. Finckh, U. et al. Variable expression of familial Alzheimer disease associated with presenilin 2 mutation M239l. Neurology 54, 2006-2008 (2000).

33. Giovagnoli, A. R., Marcon, G., Giaccone, G., Confaloni, A. M. \& Tagliavini, F. Cognitive deficits in familial Alzheimer's disease associated with M239V mutation of presenilin 2. Dement. Geriatr. Cogn. Disord. 22, 238-243 (2006).

34. Jiao, B. et al. Mutational analysis in early-onset familial Alzheimer's disease in Mainland China. Neurobiol. Aging 35, 1957.e1-1957.e6 (2014).

35. De Jonghe, C. Pathogenic APP mutations near the gamma-secretase cleavage site differentially affect Abeta secretion and APP C-terminal fragment stability. Hum. Mol. Genet. 10, 1665-1671 (2001).

36. Murrell, J. R., Hake, A. M., Quaid, K. A., Farlow, M. R. \& Ghetti, B. Early-onset Alzheimer disease caused by a new mutation (V717L) in the amyloid precursor protein gene. Arch. Neurol. 57, 885-887 (2000).

37. Ajroud-Driss, $S$. et al. Mutation in the novel nuclear-encoded mitochondria protein CHCHD10 in a family with autosomal dominant mitochondrial myopathy. Neurogenetics 16, 1-9 (2015).
38. Zhang, M. et al. Mutation analysis ofCHCHD10 in different neurodegenerative diseases. Brain 138, e380-e380 (2015).

39. Bannwarth, S. et al. A mitochondrial origin for frontotemporal dementia and amyotrophic lateral sclerosis through CHCHD10 involvement. Brain 137, 2329-2345 (2014).

40. Auranen, M. et al. CHCHD10 variant p.(Gly66Val) causes axonal Charcot-MarieTooth disease. Neurol. Genet. 1, e1 (2015).

41. Xiao, T. et al. Identification of CHCHD10 mutation in Chinese patients with Alzheimer disease. Mol. Neurobiol. 54, 5243-5247 (2016).

42. Hara, K. et al. Association of HTRA1 mutations and familial ischemic cerebral small-vessel disease. N. Engl. J. Med. 360, 1729-1739 (2009).

43. Verdura, E. et al. Heterozygous HTRA1 mutations are associated with autosomal dominant cerebral small vessel disease. Brain 138, 2347-2358 (2015).

44. Nozaki, $\mathrm{H}$. et al. Distinct molecular mechanisms of HTRA1 mutants in manifesting heterozygotes with CARASIL. Neurology 86, 1964-1974 (2016).

45. Di Donato, I. et al. Heterozygous mutations of HTRA1 gene in patients with familial cerebral small vessel disease. CNS Neurosci. Ther. 23, 759-765 (2017).

46. Petkau, T. L. \& Leavitt, B. R. Progranulin in neurodegenerative disease. Trends Neurosci. 37, 388-398 (2014).

47. Balendra, R. \& Isaacs, A. M. C9orf72-mediated ALS and FTD: multiple pathways to disease. Nat. Rev. Neurol. 14, 544-558 (2018).

48. Poorkaj, P. et al. Tau is a candidate gene for chromosome 17 frontotemporal dementia. Ann. Neurol. 43, 815-825 (1998)

49. Burrell, J. R., Kiernan, M. C., Vucic, S. \& Hodges, J. R. Motor Neuron dysfunction in frontotemporal dementia. Brain 134, 2582-2594 (2011).

50. Van Langenhove, T. et al. Predicting development of amyotrophic lateral sclerosis in frontotemporal dementia. J. Alzheimer's Dis. 58, 163-170 (2017).

51. Lomen-Hoerth. C., Anderson, T. \& Miller, B. The overlap of amyotrophic lateral sclerosis and frontotemporal dementia. Neurology 59, 1077-1079 (2002).

52. Ringholz, G. M. et al. Prevalence and patterns of cognitive impairment in sporadic ALS. Neurology 65, 586-590 (2005).

53. Watts, G. D. et al. Inclusion body myopathy associated with Paget disease of bone and frontotemporal dementia is caused by mutant valosin-containing protein Nat. Genet. 36, 377-381 (2004).

54. Al-Obeidi, E. et al. Genotype-phenotype study in patients with valosin-containing protein mutations associated with multisystem proteinopathy. Clin. Genet. 93, 119-125 (2018)

55. Blacker, D. et al. ApoE-4 and age at onset of Alzheimer's disease: the NIMH genetics initiative. Neurology 48, 139-147 (1997).

56. Corder, E. H. et al. Gene dose of apolipoprotein E type 4 allele and the risk of Alzheimer's disease in late onset families. Science 261, 921-923 (1993).

57. Li, H. \& Durbin, R. Fast and accurate long-read alignment with Burrows-Wheeler transform. Bioinformatics 26, 589-595 (2010).

58. McKenna, A. et al. The genome analysis toolkit: a MapReduce framework for analyzing next-generation DNA sequencing data. Genome Res. 20, 1297-1303 (2010).

59. Wang, K., Li, M. \& Hakonarson, H. ANNOVAR: functional annotation of genetic variants from high-throughput sequencing data. Nucleic Acids Res. 38, e164-e164 (2010).

60. den Dunnen, J. T. et al. HGVS recommendations for the description of sequence variants: 2016 update. Hum. Mutat. 37, 564-569 (2016)

61. DeJesus-Hernandez, M. et al. Expanded GGGGCC hexanucleotide repeat in noncoding region of C9ORF72 causes chromosome 9p-linked FTD and ALS Neuron 72, 245-256 (2011)

62. Bean, L. \& Bayrak-Toydemir, P. American College of Medical Genetics and Genomics Standards and Guidelines for Clinical Genetics Laboratories, 2014 edition: technical standards and guidelines for Huntington disease. Genet Med. 16, e2-e2 (2014).

63. Hooli, B. V. et al. Rare autosomal copy number variations in early-onset familial Alzheimer's disease. Mol. Psychiatry 19, 676-681 (2014).

64. Alzheimer's Disease Collaborative Group. The structure of the presenilin 1 (S182) gene and identification of six novel mutations in early onset AD families. Nat. Genet. 11, 219-222 (1995).

65. Qiu, Q. et al. A novel PSEN1 M139L mutation found in a Chinese pedigree with early-onset Alzheimer's disease increases $A \beta 42 / A \beta 40$ ratio. J. Alzheimer Dis. 69, 199-212 (2019)

66. Koriath, C. et al. Predictors for a dementia gene mutation based on gene-panel next-generation sequencing of a large dementia referral series. Mol Psychiatry. 25, 3399-3412 (2020).

67. Jin, S. C. et al. Pooled-DNA sequencing identifies novel causative variants in PSEN1, GRN and MAPT in a clinical early-onset and familial Alzheimer's disease Ibero-American cohort. Alzheimers Res. Ther. 4, 34-34 (2012).

68. Church, A. et al. A novel presenilin 1 mutation, I202F occurring at a previously predicted pathogenic site causing autosomal dominant Alzheimer's disease. Neurobiol. Aging 32, 556.e1-556.e2 (2011). 
69. Raux, G. Molecular diagnosis of autosomal dominant early onset Alzheimer's disease: an update. J. Med. Genet. 42, 793-795 (2005).

70. Coleman, P., Kurlan, R., Crook, R., Werner, J. \& Hardy, J. A new presenilin Alzheimer's disease case confirms the helical alignment of pathogenic mutations in transmembrane domain 5. Neurosci. Lett. 364, 139-140 (2004).

71. Houlden, $\mathrm{H}$. et al. A novel presenilin mutation (M233V) causing very early onset Alzheimer's disease with Lewy bodies. Neurosci. Lett. 313, 93-95 (2001).

72. Campion, D. et al. Mutations of the presenilin I gene in families with early-onset Alzheimer's disease. Hum. Mol. Genet. 4, 2373-2377 (1995).

73. Gomez-Isla, T. et al. A novel presenilin-1 mutation: increased beta-amyloid and neurofibrillary changes. Ann. Neurol. 41, 809-813 (1997).

74. Aldudo, J., Bullido, M. J., Arbizu, T., Oliva, R. \& Valdivieso, F. Identification of a novel mutation (Leu282Arg) of the human presenilin 1 gene in Alzheimer's disease. Neurosci. Lett. 240, 174-176 (1998).

75. Ikeda, M. et al. The clinical phenotype of two missense mutations in the presenilin I gene in Japanese patients. Ann. Neurol. 40, 912-917 (1996).

76. Campion, D. et al. A large pedigree with early-onset Alzheimer's disease: Clinical, neuropathologic, and genetic characterization. Neurology 45, 80-85 (1995).

77. Rogaev, E. I. et al. Familial Alzheimer's disease in kindreds with missense mutations in a gene on chromosome 1 related to the Alzheimer's disease type 3 gene. Nature 376, 775-778 (1995).

78. Ancolio, K. et al. Unusual phenotypic alteration of beta amyloid precursor protein (betaAPP) maturation by a new Val-715 $\rightarrow$ Met betaAPP-770 mutation responsible for probable early-onset Alzheimer's disease. Proc. Natl Acad. Sci. USA 96, 4119-4124 (1999).

79. Goate, A. Segregation of a missense mutation in the amyloid precursor protein gene with familial Alzheimer's disease. Nature 6311, 704-706 (1991).

80. Delisle, M. et al. A mutation at codon 279 (N279K) in exon 10 of the Tau gene causes a tauopathy with dementia and supranuclear palsy. Acta Neuropathol. 98, 62-77 (1999).

81. Guven, G. et al. Mutation frequency of the major frontotemporal dementia genes, MAPT, GRN and C9ORF72 in a Turkish cohort of dementia patients. PLOS ONE 11, e0162592 (2016).

82. Piaceri, I. et al. Novel GRN mutations in Alzheimer's disease and frontotemporal lobar degeneration. J. Alzheimer Dis. 62, 1683-1689 (2018).

83. Zhang, W., Xie, F. \& Lu, P. Two novel heterozygous HTRA1 mutations in two pedigrees with cerebral small vessel disease families. Neurol. Sci. 39, 497-501 (2018).

84. Bersano, A. et al. Inclusion body myopathy and frontotemporal dementia caused by a novel VCP mutation. Neurobiol. Aging 30, 752-758 (2009).

\section{ACKNOWLEDGEMENTS}

We are grateful to the participation of the patients and their family members in this study. This study was supported by the National Key R\&D Program of China (Nos. 2020YFC2008500, 2017YFC0840100 and 2017YFC0840104 to L.S., No. 2018 YFC1312003 to J.W.), the National Natural Science Foundation of China (Nos.
81671075 and 81971029 to L.S., No. 82071216 to B.J., and No. 81901171 to X.L.), Hunan Innovative Province Construction Project (No. 2019SK2335 to B.T.), and the Youth Science Foundation of Xiangya Hospital (No. 2018 Q020 to X.L.).

\section{AUTHOR CONTRIBUTIONS}

B.J., H.L. and L.S. designed the study, interpreted the data, and draft the manuscript. L.G., X.X., X.L., Y.Z., L.W., Lu Z., X.W., Y.J, Q.Y. and Y.Z. participated in data acquisition and analysis. Lin Z., W.Z., J.W., X.Y., J.L., B.T. and L.S. performed the data analysis; All authors read and approved the final manuscript. B.J. and H.L. contributed equally to the manuscript.

\section{COMPETING INTERESTS}

The authors declare no competing interests.

\section{ADDITIONAL INFORMATION}

Supplementary information The online version contains supplementary material available at https://doi.org/10.1038/s41525-021-00235-3.

Correspondence and requests for materials should be addressed to L.S.

Reprints and permission information is available at http://www.nature.com/ reprints

Publisher's note Springer Nature remains neutral with regard to jurisdictional claims in published maps and institutional affiliations.

(c) Open Access This article is licensed under a Creative Commons adaptation, distribution and reproduction in any medium or format, as long as you give appropriate credit to the original author(s) and the source, provide a link to the Creative Commons license, and indicate if changes were made. The images or other third party material in this article are included in the article's Creative Commons license, unless indicated otherwise in a credit line to the material. If material is not included in the article's Creative Commons license and your intended use is not permitted by statutory regulation or exceeds the permitted use, you will need to obtain permission directly from the copyright holder. To view a copy of this license, visit http://creativecommons. org/licenses/by/4.0/.

(c) The Author(s) 2021 